\title{
Leader
}

\section{Chlamydia pneumoniae in arteries: the facts, their interpretation, and future studies}

\author{
D Taylor-Robinson, B J Thomas
}

Chlamydiae are small bacteria that, as part of their growth cycle, have an obligate intracellular existence. The small infectious or elementary bodies, about $300 \mathrm{~nm}$ in diameter, attach to and are taken into cells where they develop into larger reticulate bodies. These divide by binary fission and are converted into new elementary bodies that are released from the cell. The cycle takes 48 to 72 hours.

There are four chlamydial species: Chlamydia psittaci occurs in several different animal species; in humans it causes pneumonia occasionally and abortion rarely. $C$ pecorum is also an animal species, having no known human associations. $C$ trachomatis causes trachoma, as its name implies, and certain serovars cause genital tract disease. $C$ pneumoniae commonly causes a spectrum of lower and upper respiratory tract disease in humans. ${ }^{1}$ The first association between this microorganism and coronary heart disease came from a study in Finland, ${ }^{2}$ in which it was shown that high titres of IgG and IgA antibodies to $C$ pneumoniae occurred significantly more often in men with myocardial infarction and in those with chronic coronary heart disease than in age matched, randomly selected controls. A metaanalysis of other serological studies has upheld the association between antibody and coronary heart disease, ${ }^{3}$ and overall it seems that the relative risk of disease occurring in patients with $C$ pneumoniae antibody is twofold greater than in those without antibody.

\section{Detection of $C$ pneumoniae directly in arteries}

While the serological data have elicited some general interest in the topic, it was the unexpected finding by Alan Shor in Johannesburg that created much greater interest and a flurry of scientific activity. Using electron microscopy, he noticed chlamydia-like structures in the walls of coronary arteries obtained at necropsy. The finding of $C$ pneumoniae was confirmed using the polymerase chain reaction (PCR) in an international collaborative study ${ }^{4}$ and this was followed by further confirmation. ${ }^{5}$ There have now been at least 21 other publications $^{6-27}$ (table 1) on studies in the United States, Japan, the United Kingdom, Finland, Mexico, Italy, Germany, Canada, and South Africa which testify to the existence of $C$ pneumoniae organisms in the walls of athero- matous coronary arteries and also in other major arteries. The organisms have been found in the vessels of Europids in several countries, as well as in Japanese, Indians, Alaskan Indians, and Eskimos. There has been only one study - in Brooklyn, New York, reported first in 1994 and more fully in $1996^{15}$ - in which there was failure to detect $C$ pneumoniae in coronary atheromatous tissue, despite the ability to detect it in respiratory infections that occurred frequently in the population.

\section{Chlamydial detection methods}

Of the techniques available to detect $C$ pneumoniae, PCR and immunocytochemistry have been used most often, followed by electron microscopy, culture and immunofluorescence, while enzyme immunoassay and in situ hybridisation have been used least often (table 1). All the methods have a drawback that deserves comment. The potential for spurious positive results with the PCR is well understood and needs to be guarded against. The results of immunocytochemistry and immunofluores-

Table 1 Techniques used in studies for the detection of $C$ pneumoniae in arteries

\begin{tabular}{|c|c|c|c|c|c|c|c|}
\hline \multirow[b]{2}{*}{ Reference } & \multicolumn{7}{|c|}{ Techniques used } \\
\hline & $P C R$ & $I C C$ & $E M$ & CULT & $I M F$ & $E I A$ & ISH \\
\hline 4 & & + & + & & & & \\
\hline 5 & + & + & + & & & & \\
\hline 6 & & + & & & & & \\
\hline 7 & + & + & + & & & & \\
\hline 8 & + & + & & & & & \\
\hline 9 & + & + & & & & & \\
\hline 10 & + & & & & & + & \\
\hline 11 & + & + & & & & & \\
\hline 12 & + & + & & & & & \\
\hline 13 & + & + & + & + & & & + \\
\hline 14 & & & & & + & & \\
\hline 15 & + & & + & + & & & \\
\hline 16 & + & & & & + & & \\
\hline 17 & + & & & & & & \\
\hline 18 & & & & + & & & \\
\hline 19 & + & + & & & & & \\
\hline 20 & + & + & + & + & & & \\
\hline 21 & + & + & & & & & \\
\hline 22 & & & & & + & & \\
\hline 23 & + & + & & & & & \\
\hline 24 & + & & & + & & & \\
\hline 25 & & + & & & & & \\
\hline 26 & + & & & & & & \\
\hline \multirow[t]{2}{*}{27} & + & + & + & & & & \\
\hline & 18 & 15 & 7 & 5 & 3 & 1 & 1 \\
\hline
\end{tabular}

CULT, culture; EIA, enzyme immunoassay; EM, electronmicroscopy; ICC, immunocytochemistry; IMF, immunofluorescence; ISH, in situ hybridisation; PCR, polymerase chain reaction. 
Table 2 Number (\%) of various arteries in which $C$ pneumoniae was detected in 23 studies

\begin{tabular}{llll}
\hline Artery examined & $\begin{array}{l}\text { No of } \\
\text { studies }\end{array}$ & $\begin{array}{l}\text { No of specimens } \\
\text { tested }\end{array}$ & $\begin{array}{l}\text { No (\%) } \\
\text { positive }\end{array}$ \\
\hline Coronary & 12 & 559 & $216(39)$ \\
Aorta (ascending) & 1 & 49 & $8(16)$ \\
Aorta (abdominal) & 6 & 143 & $67(47)$ \\
Aortic valve & 1 & 35 & $17(49)$ \\
Cerebral & 1 & 2 & 0 \\
Carotid & 4 & 133 & $94(71)$ \\
Pulmonary & 2 & 43 & $6(14)$ \\
Renal & 1 & 1 & 0 \\
Iliac & 2 & 15 & $8(53)$ \\
Femoral & 3 & 24 & $5(21)$ \\
Popliteal & 2 & 22 & $8(36)$ \\
Total & & $\mathbf{1 0 2 6}$ & $\mathbf{4 2 9}(\mathbf{4 2})$ \\
\hline
\end{tabular}

cence are determined subjectively and tight controlling is also required. Strong immunoperoxidase staining of peroxide-producing granules in polymorphonuclear and other inflammatory cells in atheromatous lesions is an undoubted problem not mentioned in publications. Positive staining of such bodies in atheromatous lesions but not in control tissues without inflammatory cells can lead to a false impression. Immunofluorescence for $C$ pneumoniae is not as straightforward as for $C$ trachomatis because of variation in the morphology of the elementary bodies. Results based entirely on such subjective methods are of concern, especially if the proportion of positive results is high. Greater assurance is given if there is support from the use of another technique. However, this approach may also raise questions. For example, in one study, ${ }^{19}$ of 13 positive results, five were by PCR only, seven by immunocytochemistry only, and only one by both PCR and immunocytochemistry. Such lack of concordance when examining the same tissue is of concern. Culture for $C$ pneumoniae usually requires multiple blind passages which in itself can lead to spurious positive results, but the organisms have apparently been isolated from the coronary arteries of a few subjects, ${ }^{13} 1824$ indicating that they are viable in at least some cases. Indeed, the overall results of the various studies, despite the caveats, must lead to the conclusion that $C$ pneumoniae exists in atheromatous tissue and in similar proportions of the different vessels examined (table 2). Thus of 1026 specimens examined, 429 $(42 \%)$ have been considered positive. Whether a larger proportion of arteries would have been $C$ pneumoniae positive if more tissue had been examined is a moot point.

\section{Specificity of the results of chlamydial detection tests}

The question of specificity may be considered from three points of view.

SPECIFICITY FOR C PNEUMONIAE

It is known already that $C$ pneumoniae is not specific for the atheromatous lesion since cytomegalovirus $(\mathrm{CMV})^{28}$ and herpes simplex virus type 1 (HSV-1 ${ }^{29}$ have also been found. There is a suggestion, however, that $C$ pneumoniae may be dominant. Thus, Chiu et al, ${ }^{25}$ using an immunocytochemical method with specific antisera, found that of 76 carotid specimens, 54 $(71 \%)$ were positive for $C$ pneumoniae, 27
(35.5\%) for CMV, and eight (10.5\%) for HSV-1. A single microorganism was present in $35(46 \%)$ of the specimens, two microorganisms in $18(24 \%)$, and all three microorganisms in six (8\%). The involvement of Helicobacter pylori would, at least, seem to be weak. There is no evidence that this microorganism is involved directly, and a meta-analysis of 18 serological studies has not supported an active role. ${ }^{30}$

$C$ trachomatis is known to disseminate occasionally from the genital tract to joints ${ }^{31}$ and it therefore also has an opportunity to invade blood vessels. Lack of reports that it does so may be because it has failed to invade or because it has not been sought. Oro-dental bacteria also disseminate haematogenously and their involvement in atherogenesis has been postulated. ${ }^{32}$ In addition, it has been pointed out $^{33}$ that Mycoplasma pneumoniae might be as good a candidate as $C$ pneumoniae for vessel invasion because its epidemiological behaviour and its ability to produce chronic sequelae following respiratory infection are similar. There is a precedent for mycoplasmal involvement of arteries, $M$ gallisepticum having tropism for such vessels in turkeys. ${ }^{34}$ Furthermore in one study, ${ }^{16}$ of 19 arterial specimens that were positive for $C$ pneumoniae, one was also positive for $\mathrm{M}$ fermentans by a PCR assay. While lack of specificity, even if found to be greater than appreciated currently, does not necessarily negate the importance of $C$ pneumoniae, it does mean that its role as a putative agent in atherogenesis may not be overriding.

SPECIFICITY FOR ARTERIES

The second point is whether $C$ pneumoniae is specific for arteries. Current data indicate that this is not so. There is evidence that $C$ pneumoniae may be detected in some veins ${ }^{16}$ and Thomas BJ, Taylor-Robinson D, unpublished data), in cardiac muscle, ${ }^{26}$ and in stenotic valves. ${ }^{21}$ Furthermore, it has been detected in peripheral blood monocytes ${ }^{24}$ and reported in serum of subjects who have experienced a coronary thrombosis. ${ }^{35}$ The latter seems remarkable and needs to be confirmed, but the occurrence of $C$ pneumoniae in either serum or monocytes would explain the finding of the microorganism in samples of liver and spleen, ${ }^{19}$ in cardiac muscle, and even in arteries. This is an important issue because washing specimens before DNA extraction, even if it were undertaken, may not remove all "contaminating" adherent monocytes. Another possibility for the occurrence of $C$ pneumoniae in atheromatous lesions is carriage from the respiratory tract in macrophages, the organism having been shown to infect macrophages in vitro. ${ }^{36}$

SPECIFICITY FOR ATHEROMA

The third aspect is whether $C$ pneumoniae is specific for atheromatous lesions or is also to be found in normal arteries. This brings into focus the question of controls, which may be of two sorts. The first comprises specimens without disease from age and sex matched subjects, the prevalence of $C$ pneumoniae being compared with that in specimens containing atheromatous lesions. As shown in table 3, this has been 
Table 3 Occurrence of $C$ pneumoniae in atheromatous arteries in comparison with its occurrence in non-atheromatous vessels of controls

\begin{tabular}{|c|c|c|c|c|c|c|}
\hline \multirow[b]{2}{*}{ Reference } & \multicolumn{3}{|c|}{ Atheroma group } & \multicolumn{3}{|l|}{ Control group } \\
\hline & Age (years) & $\begin{array}{l}\text { No of } \\
\text { specimens }\end{array}$ & $\begin{array}{l}\text { No positive } \\
(\%)\end{array}$ & Age (years) & $\begin{array}{l}\text { No of } \\
\text { specimens }\end{array}$ & $\begin{array}{l}\text { No positive } \\
(\%)\end{array}$ \\
\hline 5 & $<30-83$ & 36 & $20(55)$ & $\begin{array}{l}\text { Adults } \\
\text { Child (1 v) }\end{array}$ & 10 & None \\
\hline 6 & $34-58$ & 20 & $7(35)$ & Age matched & 4 & None \\
\hline 9 & $15-24$ & 1 & 1 & $15-24$ & 21 & \\
\hline & $25-34$ & 17 & $7(41)$ & $25-34$ & 10 & None \\
\hline 11 & $65-79$ & 61 & $36(59)$ & $21-46$ & 6 & None \\
\hline 14 & $50-65$ & 90 & $66(73)$ & $50-66$ & $24^{\star}$ & $1(4)$ \\
\hline 18 & $18-80$ & 38 & $13(34)$ & $?$ & 33 & $3(9)$ \\
\hline 19 & $52-76$ & 12 & $12(100)$ & $45-73$ & 12 & None \\
\hline 23 & $36-82$ & 39 & $11(28)$ & $?$ & 8 & None \\
\hline 25 & $37-85$ & 76 & $54(71)$ & $?$ & 20 & None \\
\hline 27 & $20-86$ & 24 & $17(71)$ & $22-75$ & 11 & $1(9)$ \\
\hline Total & & 414 & 244 (59) & & 160 & $5(3.1)$ \\
\hline
\end{tabular}

$\star 12$ diffusely diseased. concurrent reduction in myofilaments and an accumulation of cytoplasmic lipid.

\section{The role of $C$ pneumoniae in} atherogenesis

Although there is accumulating evidence that $C$ pneumoniae is associated with atheromatous lesions, it is difficult to resolve whether it is a primary cause of disease or whether it is a secondary invader, and if the latter, whether it behaves innocently or aggressively. From a theoretical and in vitro standpoint, the ability of infectious agents, including $C$ pneumoniae, to affect vascular cell walls directly and indirectly is known and has been discussed..$^{38}$ In vivo, if $C$ pneumoniae organisms were to be found only in the well advanced lesions of elderly subjects then it would be reasonable to assume that they were behaving as secondary invaders. However, this is not the case. Although examination of tissues from young persons less than 30 years of age has been undertaken in only five studies, in one of these $C$ pneumoniae was found in vascular tissues of subjects 15 years of age or older, but not younger. ${ }^{26}$ Histologically, only one of three PCR positive artery specimens from 15 year old persons showed lipid streaks. This poses a dilemma because it could be argued that the organisms were not associated with the lesions or that they were present ahead of the development of lesions and were involved in their initiation. The detection of $C$ pneumoniae in young subjects suggests the possibility that $C$ pneumoniae has a primary aetiological role in atherogenesis but it does not prove it. Further studies in the young and further development and use of animal models ${ }^{39}{ }^{40}$ may be helpful in resolving the "chicken or egg" conundrum. Of course, initiation or secondary invader roles are not mutually exclusive. A role as a secondary invader rather than as an innocent bystander cannot be discounted, particularly in view of the ability of $C$ trachomatis to cause chronic sequelae following primary infection. An approach to defining the role of $C$ pneumoniae is to give antichlamydial antibiotics and determine whether this diminishes the occurrence of life threatening events attributed to atherosclerosis. Two studies have already been undertaken. ${ }^{41}{ }^{42}$ Although a significant positive effect has been reported in each, both were inadequate in terms of the numbers of subjects involved and the duration of treatment. Other problems associated with such studies, and further antibiotic and other studies that might be helpful in resolving some of the issues relating to $C$ pneumoniae in atherosclerosis, are outlined below.

\section{Further studies}

Several investigations of various aspects of the topic would seem to be worthwhile.

- Specificity of C pneumoniae-Examination of tissues for various other microorganisms. Apart from those known to be present- that is, CMV and HSV-1-oro-dental bacteria and $M$ pneumoniae, in particular, need to be investigated.

- Specificity for arteries -Examination of veins from various anatomical sites to determine cle cells. They were found only in areas of tissue damage, which consisted of vacuolation of the cytoplasm of smooth muscle cells with a 
whether $C$ pneumoniae positivity is associated with lesions of any kind; this to include great saphenous veins before and after use for coronary bypass surgery.

- Specificity for atheroma-(a) Internal mammarary arteries are not regarded as having much atherosclerotic change and are used for coronary artery bypass surgery; nevertheless, they have some change. Comparing the occurrence of $C$ pneumoniae in such lesions with that in adjacent areas without change would be easier to accomplish than attempting to do this in arteries grossly affected by atheroma. (b) Examination of arteries showing evidence of arteritis-for example temporal arteritisrather than atheroma has not been reported and would seem worthwhile.

- Aetiology of atherogenesis-(a) Apart from the development of animal models, continued studies of arterial tissues from young subjects are required. Detection of $C$ pneumoniae should be combined with histology and specific antibody measurement, in attempts to answer the question of the extent to which the microorganism is associated with early atheromatous change and the extent to which antibody in the young is predictive of $C$ pneumoniae in arteries. (b) Quantification of $C$ pneumoniae in atheromatous lesions is needed to determine any relation between antigen load and the severity of lesions. It is feasible that the antigen load will be found to be greater in early, mild lesions than in more advanced severe lesions, an analogy being the infrequent detection of $C$ trachomatis in chronic trachoma. $^{43}$

- Effect of antibiotic administration, also used to define the aetiology of atherogenesis-(a) The approach has been to take the usual risk factors into account and give an antichlamydial antibiotic on a double blind, placebo controlled basis to subjects who have already survived life threatening events. The results of two such secondary prevention trials ${ }^{41}{ }^{42}$ have indicated that the treatment, azithromycin in one $\mathrm{e}^{41}$ and roxithromycin in the other, ${ }^{42}$ has prevented further such events more than placebo. These trials, particularly the former, have been criticised ${ }^{44}$ for the small number of participants studied and inadequate dosage. Apart from correcting these faults in future trials, it would also be advantageous to have a relatively noninvasive means of determining whether or not $C$ pneumoniae existed in the arteries of the patients in question. On an individual basis, measurement of antibody is probably of no value in this endeavour. Although certain antigens of $C$ pneumoniae, defined by immunoblotting, have been linked to cardiovascular risk, ${ }^{45}$ the detection of $C p^{n-}$ eumoniae in peripheral blood monocytes ${ }^{24}$ has raised the possibility that this might be a marker of arterial involvement, and this needs further evaluation. It would be a formidable undertaking to obtain coronary artery material to seek $C$ pneumoniae and concurrently try to correlate results with the occurrence of $C$ pneumoniae in peripheral blood monocytes. More feasible would be an attempt to correlate $C$ pneumoniae in monocytes with the degree of atherosclerosis judged by angiography. Furthermore, as a preliminary to or in parallel with extensive antibiotic controlled trials, angiographytogether with examination of monocytes for $C$ pneumoniae - could be followed by placebo controlled antibiotic treatment. Subsequently, monocytes could be examined for $C$ pneumoniae clearance and angiography undertaken to determine whether any diminution in atheromatous changes had occurred. Tissues from treated and untreated subjects who progressed to bypass surgery could be examined for $C$ pneumoniae to determine whether treatment had cleared $C$ pneumoniae from the tissues. (b) Similarly, it would seem worthwhile determining the effect of antibiotic treatment in relation to carotid artery changes. (c) Further extensive antibiotic trials on subjects who have or have not experienced life threatening events associated with coronary heart disease are inevitable, despite the caveats about the role of $C$ pneumoniae expressed here. These trials could include efforts to determine whether there is a delay in the time to restenosis in subjects treated with antibiotics following coronary bypass surgery. Some investigators may consider that such trials are a definitive way of determining the role of $C$ pneumoniae. It must be recognised, however, that a broad spectrum antibiotic, such as azithromycin, is not specific for $C$ pneumoniae, and any beneficial effect cannot necessarily be assumed to be due to the clearance of $C$ pneumoniae, even if the studies outlined above show that this is feasible. Defining the role of $C$ pneumoniae in such trials might be helped by treating subjects who have high titres of $C$ pneumoniae specific antibody and comparing the results with those obtained by treating those without antibody, on the assumption that only a small proportion of the latter group had been infected arterially. If $C$ pneumoniae were to be involved, a greater remedial effect would be expected in the former group. That lack of antibody on a group rather than an individual basis means lack of arterial infection is likely to remain an assumption, as it is not possible to know that all arteries are free of $C$ pneumoniae. The most that could be achieved would be to show that lack of antibody was or was not related to failure to detect $C$ pneumoniae in peripheral blood monocytes. In turn, this would be of value if the relation between monocyte chlamydial positivity and the occurrence of $C$ pneumoniae in arteries were known. While some may consider that defining the exact role of $C$ pneumoniae in this way is a purely academic exercise, particularly since obtaining subjects without antibody will always be difficult because of the widespread distribution of $C$ pneumoniae and the common occurrence of antibody to it, defining the cause of disease should never be discouraged. An alternative approach 
would be to undertake placebo controlled antibiotic trials, not using the presence or absence of antibody/C pneumoniae positive or negative monocytes as inclusion criteria, but nevertheless assessing these factors and considering their relation to the outcome of the trials subsequently. (d) The role of cell mediated immunity and autoimmunity, through heat shock protein cross reactivity, in the pathogenesis of chronic chlamydial infections such as trachoma and pelvic inflammatory disease is recognised, although the mechanisms are not understood in detail. ${ }^{46}$ Seeking such responses, in the context of $C$ pneumoniae and atheroma formation or enhancement, provides another avenue of exploration. (e) The ultimate step would be the administration of a specific $C$ pneumoniae vaccine, particularly to young subjects, followed by long term surveillance. The impetus for this might come from antibiotic trials if they prove successful in diminishing the number of life threatening events, and even if they did not show the specific involvement of $C$ pneumoniae. What is not required in the future is a succession of studies proclaiming the existence of $C$ pneumoniae organisms in arteries. This is known. Greater ingenuity is needed to determine the importance of their existence in arteries.

1 Grayston JT. Infections caused by Chlamydia pneumoniae strain TWAR. Clin Infect Dis 1992;15:757-63.

2 Saikku P, Leinonen M, Mattila K, et al. Serological evidence of an association of novel Chlamydia, TWAR, with chronic coronary heart disease and acute myocardial infarction. Lancet 1988;ii:983-6.

3 Danesh J, Collins R, Peto R. Chronic infections and coronary heart disease: is there a link? Lancet 1997;350:378-9.

4 Shor A, Kuo C-C, Patton DL. Detection of Chlamydia pneumoniae in coronary arterial fatty streaks and atheromatous plaques. $S$ Afr Med $\mathcal{F} 1992 ; 82: 158-61$.

5 Kuo C-C, Shor A, Campbell LA, et al. Demonstration of Chlamydia pneumoniae in atherosclerotic lesions of coronary arteries. F Infect Dis 1993;167:841-9.

6 Kuo C-C, Gown AM, Benditt EP, et al. Detection of Chlamydia pneumoniae in aortic lesions of atherosclerosis by immunocytochemical stain. Arterioscler Thromb 1993 13:1501-4.

7 Campbell LA, O’Brien ER, Cappuccio AL, et al. Detection of Chlamydia pneumoniae TWAR in human coronary atherectomy tissues. F Infect Dis 1995;172:585-8.

8 Davidson M, Kuo C-C, Middaugh JP, et al. Chlamydia pneumoniae (TWAR) in Alaskan natives with coronary pneumoniae (TWAR) in Alaskan natives W.

9 Kuo C-C, Grayston JT, Campbell LA, et al. Chlamydia pneumoniae (TWAR) in coronary arteries of young adults (15-34 years old). Proc Natl Acad Sci USA 1995;92:691114.

10 Varghese PJ, Gaydos CA, Arumugham SB, et al. Demonstration of Chlamydia pneumoniae in coronary atherom specimens from young patients with normal cholesterol from the Southern part of India [abstr]. Clin Infect Dis 1995;21:728.

11 Grayston JT, Kuo C-C Coulson AS, et al. Chlamydia pneumoniae (TWAR) in atherosclerosis of the carotid artery. Circulation 1995;92:3397-400.

12 Ouchi K, Fujii B, Kanamato Y, et al. Detection of Chlamydia pneumoniae in atherosclerotic lesions of coronary dia pneumoniae in atherosclerotic lesions of coronary arteries and large arteries [abstr]. 35th Interscience Confer-
ence on Antimicrobial Agents and Chemotherapy. San ence on Antimicrobial Agents and Chemotherapy. S

13 Ramirez JA, the Chlamydia pneumoniae/Atherosclerosis Study Group. Isolation of Chlamydia pneumoniae from the coronary artery of a patient with coronary atherosclerosis. Ann Intern Med 1996;125:979-82.

14 Muhlestein JB, Hammond EH, Carlquist JF, et al. Increased incidence of Chlamydia species within the coronary arteries of patients with symptomatic atherosclerotic versus other forms of cardiovascular disease. $7 \mathrm{Am}$ Coll Cardiol 1996;27:1555-61.

15 Weiss SM, Roblin PM, Gaydos CA, et al. Failure to detect Chlamydia pneumoniae in coronary atheromas of patients
undergoing atherectomy. F Infect Dis 1996;173:957-62.

16 Ong G, Thomas BJ, Mansfield AO, et al. Detection and widespread distribution of Chlamydia pneumoniae in the vascular system and its possible implications. 7 Clin Pathol 1996;49:102-6.

17 Blasi F, Denti F, Erba M, et al. Detection of Chlamydia pneumoniae but not Helicobacter pylori in atherosclerotic plaques of aortic aneurysms. F Clin Microbiol 1996;34: 2766-9.

18 Jackson LA, Campbell LA, Kuo C-C, et al. Isolation of Chlamydia pneumoniae from a carotid endarterectomy specimen. F Infect Dis 1997;176:292-5.

19 Jackson LA, Campbell LA, Schmidt RA, et al. Specificity of detection of Chlamydia pneumoniae in cardiovascular atheroma. Am f Pathol 1997;150:1785-90.

20 Juvonen J, Juvonen T, Laurila A, et al. Demonstration of Chlamydia pneumoniae in the walls of abdominal aortic aneurysms. F Vasc Surg 1997;25:499-505.

21 Juvonen J, Laurila A, Juvonen T, et al. Detection of Chlamydia pneumoniae in human nonrheumatic stenotic aortic valves. $\mathcal{F}$ Am Coll Cardiol 1997;29:1054-9.

22 Gloria-Breceda F, Meaney-Mendiolea E, Valero-Elizondo $\mathrm{G}$, et al. The relationship between Chlamydia pneumoniae and atherosclerotic lesions of the aorta. Arch Inst Cardiol Mex 1997;67:17-23.

23 Kuo C-C, Coulson AS, Campbell LA, et al. Detection of Chlamydia pneumoniae in atherosclerotic plaques in the walls of arteries of lower extremities from patients undergoing bypass operation for arterial obstruction. $\mathcal{F}$ Vasc Surg 1997;26:29-31.

24 Maass M, Krause E, Krüger S, et al. Coronary arteries harbour viable Chlamydia pneumoniae [abstr]. Clin Microbiol Infect 1997;3(suppl 2):136.

25 Chiu B, Viira E, Tucker W, et al. Chlamydia pneumoniae, cytomegalovirus, and Herpes simplex virus in atherosclerosis of the carotid artery. Circulation 1997;96:2144-8.

26 Taylor-Robinson D, Ong G, Thomas BJ, et al. Chlamydia pneumoniae in vascular tissues from heart transplant donors. Lancet 1998;351:1255.

27 Shor A, Phillips JI, Ong G, et al. Chlamydia pneumoniae in atheroma; consideration of criteria for causality. F Clin Pathol [in press].

28 Melnick JL, Hu C, Burek J, et al. Cytomegalovirus DNA in arterial walls of patients with atherosclerosis. $\mathcal{F}$ Med Virol 1994;42:170-4

29 Benditt EP, Barrett T, McDougall JK. Viruses in the tiology of atherosclerosis. Proc Natl Acad Sci USA 1983;80:6386-9.

30 Danesh J, Peto R. Risk factors for coronary heart disease and infection with Helicobacter pylori: metanalysis of 18 studies. BMF 1998;316:1130-2

31 Keat A, Thomas B, Dixey J, et al. Chlamydia trachomatis and reactive arthritis: the missing link. Lancet 1987; 72-4.

32 Beck J, Garcia R, Heiss G, et al. Periodontal disease and cardiovascular disease. F Periodontol 1996;67:1123-37.

33 Taylor-Robinson D. Chlamydia pneumoniae infection and coronary heart disease. Trials should assess whether antibiotics eliminate organism from atherosclerotic lesions. BMF 1997;315:1538.

34 Clyde WA, Thomas L. Tropism of Mycoplasma gallisepticum for arterial walls. Proc Natl Acad Sci USA 1973;70: $1545-9$

35 Naidu BR, Ngeow YF, Kannan P, et al. Evidence of Chlamydia pneumoniae infection obtained by the polymerase chain reaction (PCR) in patients with acute myocardial infarction and coronary heart disease. F Infect 1997;35: 199-200.

36 Saikku P. Chlamydia pneumoniae and atherosclerosis-an update. Scand F Infect Dis 1997;104(suppl):53-6.

37 Hajjar DP, Nicholson AC. Atherosclerosis. Am Sci 1995;83: 460-7.

38 Libby P, Egan D, Skarlatos S. Roles of infectious agents in atherosclerosis and restenosis. An assessment of the evidence and need for future research. Circulation 1997;96: 4095-103.

39 Moazed TC, Kuo C, Grayston JT, et al. Murine models of Chlamydia pneumoniae infection and atherosclerosis. $\mathcal{F}$ Infect Dis 1997;175:883-90.

40 Fong IW, Chiu B, Viira E, et al. Rabbit model for Chlamydia pneumoniae infection. $\mathcal{F}$ Clin Microbiol 1997;35:48-52.

41 Gupta S, Leatham EW, Carrington D, et al. Elevated Chlamydia pneumoniae antibodies, cardiovascular events, and azithromycin in male survivors of myocardial infarction. Circulation 1997;96:404-7.

42 Gurfinkel E, Bozovich G, Daroca A, et al, for the ROXIS Study Group. Randomised trial of roxithromycin in non-Q-wave coronary syndromes: ROXIS pilot study. Lancet 1997;350:404-7.

43 Taylor-Robinson D, Mabey DCW, Treharne JD. Chlamydial infections. In: Weatherall DJ, Leadingham JGG, Warrell DA, eds. Oxford textbook of medicine, 3rd ed, vol 1 . Oxford: Oxford University Press, 1996:748-59.

44 Grayston JT. Antibiotic treatment of Chlamydia pneumoniae for secondary prevention of cardiovascular events. In: Stephens RS, Byrne GI, Christiansen G, et al, eds. Chlamydial infections. Proceedings of the Ninth International Symposium on Human Chlamydial Infections, Napa, USA. San Francisco: University of California, 1998:187-90.

45 Maass M, Gieffers J. Cardiovascular disease risk from prior Chlamydia pneumoniae infection can be related to certain antigens recognised in the immunoblot profile. I Infect 1997;35:171-6.

46 Ward ME. The immunobiology and immunopathology of chlamydial infections. APMIS 1995;103:769-96. 